\title{
WG-8: A Lightweight Stream Cipher for Resource-Constrained Smart Devices
}

\author{
Xinxin Fan*, Kalikinkar Mandal and Guang Gong \\ Department of Electrical and Computer Engineering \\ University of Waterloo \\ Waterloo, Ontario, N2L 3G1, CANADA
}

Abstract

Lightweight cryptographic primitives are essential for securing pervasive embedded devices like RFID tags, smart cards, and wireless sensor nodes. In this paper, we present a lightweight stream cipher WG-8, which is tailored from the well-known Welch-Gong (WG) stream cipher family, for resource-constrained devices. WG-8 inherits the good randomness and cryptographic properties of the WG stream cipher family and is resistant to the most common attacks against stream ciphers. The software implementations of the WG-8 stream cipher on two popular low-power microcontrollers as well as the extensive comparison with other lightweight cryptography implementations highlight that in the context of securing lightweight embedded applications WG-8 has favorable performance and low energy consumption. Keywords: Lightweight stream cipher, resource-constrained smart device, cryptanalysis, efficient implementation.
Received on 29 March 2014; accepted on 23 December 2014, published on 30 January 2015
Copyright (C) 2014 Xinxin Fan et al., licensed to ICST. This is an open access article distributed under the terms of the
Creative Commons Attribution license (http: / / creativecommons. org/licenses / by/3.0/), which permits
unlimited use, distribution and reproduction in any medium so long as the original work is properly cited.

doi:10.4108/sesa.2.3.e4

\section{Introduction}

The Internet of Things (IoT) is an emerging computing and communication paradigm in which smart devices (e.g., RFID tags, smart cards, wireless sensor nodes, etc.) are linked through both wired and wireless networks to the Internet. Those smart devices interact and cooperate with each other to conduct complicated tasks such as sensing the environment, interpreting the data, and responding to events. While the IoT provides new and exciting experience for end users, it also opens up new avenues to hackers and organized crime. Recent attacks to a wide range of smart devices $[14,41]$ have emphasized that without adequate security the IoT will only become pervasive nightmare.

The challenges for deploying security solutions for smart devices are threefold: 1) The overhead (i.e., the gate count in hardware or the memory footprint in software) of security solutions should

*Corresponding author. x5fan@uwaterloo.ca be minimal due to the low-cost nature of smart devices; 2) The power consumption of security solutions should be minimal due to the low-power characteristic of smart devices; and 3) The performance of security solutions should be reasonable to support applications and end-user requirements. To address the aforementioned challenges for securing smart devices, a new research direction called lightweight cryptography has been established which focuses on designing novel cryptographic algorithms and protocols tailored for implementation in resource-constrained environments.

A host of lightweight symmetric ciphers that particularly target for resource-constrained smart devices have been proposed in the past few years. Early work focuses on optimizing hardware implementations of standardized block ciphers such as AES [18], IDEA [26] and XTEA [23]. Later on, researchers have shown how to modify a classical block cipher like DES [25] for lightweight applications. Recent proposals deal with new low-cost designs, including lightweight block ciphers 
PRESENT [5], KATAN/KTANTAN [6], PRINTcipher [24], LED [21], and Piccolo [38], lightweight stream ciphers Grain [22], Trivium [7], and MICKEY [3], as well as a lightweight hybrid cipher Hummingbird/Hummingbird-2 $[16,17]$. A good research survey about recently published lightweight cryptographic implementations can be found in [15].

In this paper we present the stream cipher WG-8, which is a lightweight variant of the well-known WG stream cipher family [31] as submitted to the eSTREAM project. WG-8 inherits good randomness properties of the WG stream cipher family such as period, balance, ideal two-level autocorrelation, ideal tuple distribution, and exact linear complexity. Moreover, WG-8 is able to resist the most common attacks against stream ciphers including algebraic attack, correlation attack, differential attack, cube attack, distinguish attack, discrete fourier transform attack, and time-memory-data tradeoff attack, thereby providing adequate security for lightweight embedded applications.

We also propose several techniques for efficient implementation of the stream cipher WG-8 on two lowpower microcontrollers, including an 8-bit microcontroller ATmega128L from Atmel and a 16-bit microcontroller MSP430 from Texas Instruments. Our experimental results show that WG-8 can achieve high throughput of $185.5 \mathrm{Kbits} / \mathrm{s}$ and $95.9 \mathrm{Kbits} / \mathrm{s}$ on the above two microcontrollers with energy efficiency of $458 \mathrm{~nJ} / \mathrm{bit}$ and $125 \mathrm{~nJ} /$ bit, respectively. When compared to other lightweight cryptography implementations in the literature, the throughput of the WG- 8 is about $2 \sim 15$ times higher and the energy consumption is around $2 \sim 220$ times smaller than those of most previous ciphers.

The remainder of this paper is organized as follows. Section 2 gives a description of the lightweight stream cipher WG-8. Subsequently, in Section 3 we analyze the security of the WG-8 against the most common attacks to stream ciphers. Section 4 describes efficient techniques for implementing the WG-8 stream cipher on low-power microcontrollers and reports our experimental results and comparisons with previous work. In Section 5 , we briefly review recent progress on efficient hardware implementation of the WG-8 stream cipher. Finally, Section 6 concludes this contribution.

\section{The Lightweight Stream Cipher WG-8}

\subsection{Preliminaries}

We define the terms and notations that will be used to describe the lightweight stream cipher WG-8 and its architecture as well as to characterize its randomness and cryptographic properties.

- $\mathbb{F}_{2}=\{0,1\}$, the Galois field with two elements 0 and 1.

- $p(x)=x^{8}+x^{4}+x^{3}+x^{2}+1$, a primitive polynomial of degree 8 over $\mathbb{F}_{2}$.

- $\mathbb{F}_{2^{8}}$, the extension field of $\mathbb{F}_{2}$ defined by the primitive polynomial $p(x)$ with $2^{8}$ elements. Each element in $\mathbb{F}_{2^{8}}$ is represented as an 8-bit binary vector. Let $\omega$ be a primitive element of $\mathbb{F}_{2^{8}}$ such that $p(\omega)=0$.

- $\operatorname{Tr}(x)=x+x^{2}+x^{2^{2}}+\cdots+x^{2^{7}}$, the trace function from $\mathbb{F}_{2^{8}} \mapsto \mathbb{F}_{2}$.

- $l(x)=x^{20}+x^{9}+x^{8}+x^{7}+x^{4}+x^{3}+x^{2}+x+\omega$, the feedback polynomial of LFSR (which is also a primitive polynomial over $\mathbb{F}_{2^{8}}$ ).

- $q(x)=x+x^{2^{3}+1}+x^{2^{6}+2^{3}+1}+x^{2^{6}-2^{3}+1}+$ $x^{2^{6}+2^{3}-1}$, a permutation polynomial over $\mathbb{F}_{2^{8}}$.

- WGP-8 $\left(x^{d}\right)=q\left(x^{d}+1\right)+1$, the WG-8 permutation with decimation $d$ from $\mathbb{F}_{2^{8}} \mapsto \mathbb{F}_{2^{8}}$, where $d$ is coprime to $2^{8}-1$.

- WGT-8 $\left(x^{d}\right)=\operatorname{Tr}\left(\mathrm{WGP}-8\left(x^{d}\right)\right)=\operatorname{Tr}\left(x^{9}+x^{37}+\right.$ $\left.x^{53}+x^{63}+x^{127}\right)$, the WG-8 transformation with decimation $d$ from $\mathbb{F}_{2^{8}} \rightarrow \mathbb{F}_{2}$, where $d$ is coprime to $2^{8}-1$.

- Polynomial basis (PB) of $\mathbb{F}_{2^{8}}$ : A polynomial basis of $\mathbb{F}_{2^{8}}$ over $\mathbb{F}_{2}$ is a basis of the form $\left\{1, \omega, \omega^{2}, \cdots, \omega^{7}\right\}$.

- Normal basis (NB) of $\mathbb{F}_{2^{8}}$ : A normal basis of $\mathbb{F}_{2^{8}}$ over $\mathbb{F}_{2}$ is a basis of the form $\left\{\theta, \theta^{2}, \cdots, \theta^{2^{7}}\right\}$, where $\theta=\omega^{5}$ (i.e., a normal element) is used in this work.

- Autocorrelation: The autocorrelation of a binary sequence with period $T$ is defined as the difference between the agreements and disagreements when the symbol 0 maps to 1 and 1 maps to -1 . If all 
the out-of-phase autocorrelation is equal to -1 , then the sequence is said to have ideal two-level autocorrelation.

- Linear span (LS): The linear span or linear complexity of a binary sequence is defined as the length of the smallest linear feedback shift register (LFSR) which generates the entire binary sequence.

- Nonlinearity: The nonlinearity of a function $f$ is defined as the minimum distance from $f$ to any affine function with the same number of variables.

- Algebraic immunity (AI): The algebraic immunity of a function $f$ is defined as the minimum degree of an annihilator Boolean function $g$ such that $g$ is equivalent to either $f$ or the complement of $f$ (i.e., $f g=0$ or $(f+1) g=0)$. In the ideal case, the algebraic immunity of a function $f$ is equal to the degree of $f$, thus making it immune to algebraic attacks.

- $\oplus$, the bitwise addition operator (i.e., XOR).

- $\otimes$, the multiplication operator over $\mathbb{F}_{2^{8}}$.

\subsection{The Description of the Stream Cipher WG-8}

WG-8 is a lightweight variant of the well-known WelchGong (WG) stream cipher family with 80-bit secret key and 80-bit initial vector (IV), which can be regarded as a nonlinear filter generator over finite field $\mathbb{F}_{2^{8}}$. The stream cipher WG-8 consists of a 20-stage LFSR with the feedback polynomial $l(x)$ followed by a WG8 transformation module with decimation $d=19$, and operates in two phases, namely an initialization phase and a running phase.

Initialization Phase. The key/IV initialization phase of the stream cipher WG-8 is shown in Figure 1.

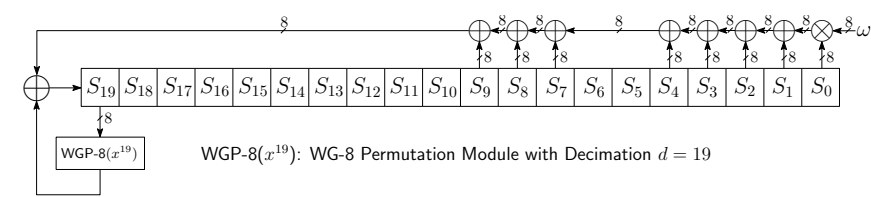

Figure 1. The Initialization Phase of the Stream Cipher WG-8
Let the 80 -bit secret key be $K=\left(K_{79}, \ldots, K_{0}\right)_{2}$, the 80 -bit IV be $I V=\left(I V_{79}, \ldots, I V_{0}\right)_{2}$, and the internal state of the LFSR be $S_{0}, \ldots, S_{19} \in \mathbb{F}_{2^{8}}$, where $S_{i}=\left(S_{i, 7}, \ldots, S_{i, 0}\right)_{2}$ for $i=0, \ldots, 19$. The key and IV initialization process is conducted as follows: $S_{2 i}=\left(K_{8 i+3}, \ldots, K_{8 i}, I V_{8 i+3}, \ldots, I V_{8 i}\right)_{2}$ and $S_{2 i+1}=$ $\left(K_{8 i+7}, \ldots, K_{8 i+4}, I V_{8 i+7}, \ldots, I V_{8 i+4}\right)_{2}$ for $i=0, \ldots, 9$.

Once the LFSR is loaded with the key and IV, the apparatus runs for 40 clock cycles. During each clock cycle, the 8-bit internal state $S_{19}$ passes through the nonlinear WG-8 permutation with decimation $d=19$ (i.e., the WGP-8 $\left(x^{19}\right)$ module) and the output is used as the feedback to update the internal state of the LFSR. The LFSR update follows the recursive relation:

$$
\begin{aligned}
& S_{k+20}=\left(\omega \otimes S_{k}\right) \oplus S_{k+1} \oplus S_{k+2} \oplus S_{k+3} \oplus S_{k+4} \oplus \\
& S_{k+7} \oplus S_{k+8} \oplus S_{k+9} \oplus \mathrm{WGP}-8\left(S_{k+19}^{19}\right), \quad 0 \leq k<40 .
\end{aligned}
$$

After the key/IV initialization phase, the stream cipher WG-8 goes into the running phase and 1-bit keystream is generated after each clock cycle.

Running Phase. The running phase of the stream cipher WG-8 is illustrated in Figure 2. During the running phase, the 8-bit internal state $S_{19}$ passes through the nonlinear WG-8 transformation with decimation $d=$ 19 (i.e., the WGT-8 $\left(x^{19}\right)$ module) and the output is the keystream. Note that the only feedback in the running phase is within the LFSR and the recursive relation for updating the LFSR is given below:

$$
\begin{aligned}
& S_{k+20}=\left(\omega \otimes S_{k}\right) \oplus S_{k+1} \oplus S_{k+2} \oplus S_{k+3} \oplus S_{k+4} \oplus \\
& S_{k+7} \oplus S_{k+8} \oplus S_{k+9}, \quad k \geq 40 .
\end{aligned}
$$

The WG-8 transformation module WGT-8 $\left(x^{19}\right)$ comprises of two sub-modules: a WG-8 permutation module WGP-8 $\left(x^{19}\right)$ followed by a trace computation module $\operatorname{Tr}(\cdot)$. While the WGP-8 $\left(x^{19}\right)$ module permutes elements over $\mathbb{F}_{2^{8}}$, the $\operatorname{Tr}(\cdot)$ module compresses an 8-bit input to 1-bit keystream.

\subsection{Randomness Properties of the WG-8 Keystream}

The keystream generated by the stream cipher WG-8 has the following desired randomness properties [8]: 


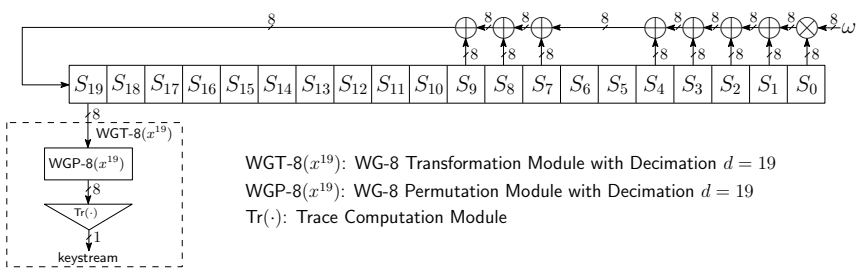

Figure 2. The Running Phase of the Stream Cipher WG-8

1. The keystream has a period of $2^{160}-1$.

2. The keystream is balanced, i.e., the number of 0's is only one less than the number of 1's in one period of the keystream.

3. The keystream is an ideal two-level autocorrelation sequence.

4. The keystream has an ideal $t$-tuple $(1 \leq t \leq 20)$ distribution, i.e., every possible output $t$-tuple is equally likely to occur in one period of the keystream.

5. The linear span of the keystream can be determined exactly, which is $2^{33.32}$.

\section{Cryptanalysis of the Stream Cipher WG-8}

In this section, we analyze the security of the stream cipher WG-8 under the context of lightweight embedded applications. Note that for security applications (e.g., encryption, authentication, etc.) with smart devices the communication sessions are typically short (i.e., a few rounds among communication entities) and the length of transmitted messages is generally quite limited (i.e., tens to hundreds of bytes) in each communication session, due to the constrained power and communication bandwidth of smart devices. As a result, it is extremely difficult, if not impossible, for an adversary collecting consecutive and long enough keystream to conduct cryptanalysis in practice for a well-designed lightweight stream cipher. The design of the stream cipher WG-8 keeps in mind the real-world embedded security applications and the appropriate selection of system parameters enables WG8 to thwart various cryptanalytic attacks in practice, as detailed in the following subsections.

\subsection{Algebraic Attack}

The algebraic attack is a powerful attack against LFSR based filtering sequence generators [11]. The goal of the algebraic attack is to form a lower degree multivariate equation by multiplying the filtering function by a low-degree multivariate polynomial. This gives an overdefined system of nonlinear equations for sufficiently many keystreams, which can be solved to recover the internal state of the LFSR. The algebraic immunity of the WGT-8 $\left(x^{19}\right)$ is equal to 4 . According to the algebraic attack, the time complexity and the data complexity for recovering the internal state of the LFSR are about $\frac{7}{64} \cdot\left(\begin{array}{c}160 \\ 4\end{array}\right)^{\log _{2} 7}=2^{66.0037}$ and $\left(\begin{array}{c}160 \\ 4\end{array}\right)=2^{24.65}$, respectively. For applying the fast algebraic attacks [10] to the stream cipher WG-8, one needs to respectively find two multivariate polynomials $g$ and $h$ of degree $e$ and $d(e<d)$ such that $f \cdot g=h$. For the WGT-8 $\left(x^{19}\right)$ and $e=1$, there does not exist a multivariate polynomial $h$ in 8 variables with degree less than 7 . Hence, in order to launch the fast algebraic attack one needs to obtain more keystream bits with a higher complexity. For lightweight embedded applications, it is hard for an attacker to obtain about $2^{24.65}$ keystream bits. Even if the attacker can get those many bits for a fixed key and IV, he needs to perform the operations with the time complexity $2^{66.0037}$, which completely defeats this attack.

\subsection{Correlation Attack}

In the correlation attack, the objective of an attacker is either to find the correlation between a keystream and an output sequence of an LFSR or to find the correlation among the keystreams [9, 28, 39]. The stream cipher WG-8 is secure against the correlation among the keystreams as it produces keystreams with 2-level autocorrelation. We now consider the fast correlation attack in which the keystream of the stream cipher is considered as a distorted version of the LFSR output. In the fast correlation attack, the linear approximation of WGT-8 $\left(x^{19}\right)$ can be used to derive a generator matrix of a linear code that can be decoded by a maximum likelihood decoding (MLD) algorithm. Letting $f(x)$ be a linear function in 8 variables, we have $\operatorname{Pr}\left(\right.$ WGT- $8\left(x^{19}\right)(x)=$ $f(x))=\frac{\left(2^{8}-108\right)}{2^{8}}=0.578125$. Applying the results of [9] for $t=3$, the amount of keystream (denoted by 
$N)$ required for the attack to be successful is given by $N \approx(k \cdot 12 \cdot \ln 2)^{\frac{1}{3}} \cdot \epsilon^{-2} \cdot 2^{\frac{160-k}{3}}$ and the decoding complexity is given by $C_{d e c}=2^{k} \cdot k \cdot \frac{2 \ln 2}{(2 \epsilon)^{6}}$, where $\epsilon=$ $\left(\operatorname{Pr}\left(\right.\right.$ WGT-8 $\left.\left.\left(x^{19}\right)=f(x)\right)-0.5\right)=0.078125$ and $k$ is the number of LFSR internal state bits recovered. If we choose a small value of $k$ (e.g., $k=7$ ), the number of bits required to launch the attack is about $2^{60.31}$, which is not possible in practice. Similarly, if we choose a large value of $k$ (e.g., $k=80$ ), the number of bits required to mount the attack is about $2^{37.15}$. However, the decoding complexity of the attack is approximately $2^{102.68}$, which is worse than the exhaustive search. Hence, the stream cipher WG-8 is secure against the fast correlation attack.

\subsection{Differential Attack}

The initialization phase in the first design of the WG stream cipher was vulnerable to the chosen IV attack [43], where an attacker can distinguish several output bits by building a distinguisher based on the differential cryptanalysis. This weakness has been fixed in the later design by placing the WG permutation module at the last position of the LFSR [31]. For the proposed stream cipher WG-8, the differential distribution of the WGP-8 $\left(x^{19}\right)$ is 8 -uniform, which provides a maximum $2^{-5}$ possibility for differential characteristic. During the initialization phase the WGP-8 $\left(x^{19}\right)$ is applied for 40 times. Thus, after the initialization phase, it would be quite hard for an attacker to distinguish the output keystream since the differentials become complex and contain most key/IV bits.

\subsection{Cube Attack}

Cube attack [13] is a generic key-recovery attack that can be applied to any cryptosystem, provided that the attacker can obtain a bit of information that can be represented by a low-degree decomposition multivariate polynomial in Algebraic Normal Form (ANF) of the secret and public variables of the target cryptosystem. Note that the nonlinearity of WGP-8 $\left(x^{19}\right)$ is 92 and the algebraic degrees of the component functions of WGP$8\left(x^{19}\right)$ are 7 . Moreover, the ANF representations of 8 component functions contain 133, 113, 146, 124, 137, 109, 122 , and 120 terms, respectively, and only the ANF of the second component contains 7 linear terms and other terms are of degree greater than or equal to 2 . In the WG-8 stream cipher, after 40 rounds of the initialization phase, the degree of the output polynomial can be very high. As a result, it would be hard for an attacker to collect low-degree relations among the secret key bits.

\subsection{Distinguishing Attack}

Recently, a distinguishing attack has been proposed against the stream cipher WG-7 [32]. Due to the small number of tap positions in the LFSR of the WG-7, the characteristic polynomial of the LFSR allows an attacker to build a distinguisher for distinguishing a keystream generated by WG-7 from a truly random keystream. For the WG-8 cipher, the characteristic polynomial of the LFSR consists of 8 tap positions and a similar distinguisher as in [32] can be built as

$$
\begin{aligned}
& F\left(S_{i}, \ldots, S_{i+4}, S_{i+7}, \ldots, S_{i+9}\right)=\mathrm{WGT}-8\left(\omega \otimes S_{i} \oplus\right. \\
& \left.S_{i+1} \oplus S_{i+2} \oplus S_{i+3} \oplus S_{i+4} \oplus S_{i+7} \oplus S_{i+8} \oplus S_{i+9}\right) \oplus \\
& \text { WGT-8 }\left(S_{i}\right) \oplus \mathrm{WGT-8}\left(S_{i+1}\right) \oplus \operatorname{WGT-8}\left(S_{i+2}\right) \oplus \\
& \text { WGT-8 }\left(S_{i+3}\right) \oplus \text { WGT-8 }\left(S_{i+4}\right) \oplus \text { WGT-8 }\left(S_{i+7}\right) \oplus \\
& \text { WGT-8 }\left(S_{i+8}\right) \oplus \operatorname{WGT-8}\left(S_{i+9}\right),
\end{aligned}
$$

which is a Boolean function in 64 variables. For the distinguisher $F$, the probability $\operatorname{Pr}(F(x)=0)=\frac{1}{2} \pm \epsilon$, where $x=\left(a_{0}, \ldots, a_{7}\right), a_{i} \in \mathbb{F}_{2^{8}}$. Note that the value of $\epsilon$ will be quite small due to a huge number of variables in the distinguisher, which requires an attacker to obtain more keystream bits for distinguishing the keystream. However, the computation of the exact value of $\epsilon$ is infeasible in this case because the number of possible values of $x$ is $2^{64}$. Hence the WG- 8 stream cipher is resistant to the distinguishing attack. Note that this type of distinguishing attacks can also be extended to the case in which a distinguisher can be built using a linear relation of a remote term of the LFSR, say $S_{\tau}$ for not large $\tau$, and the sequences addressed in a subset of tap positions of the LFSR, denoted by $I=\left\{i_{1}, \cdots, i_{t}\right\} \subset$ $\{0,1, \cdots, 19\}$. In other words, a distinguisher could be built using the linear relation $S_{\tau}=S_{i_{1}}+\cdots+S_{i_{t}}$. Since this property is controlled by the characteristic polynomial of the LFSR, it can be easily teared done by a proper selection of the characteristic polynomial of the LFSR. For our selection of the characteristic 
polynomial $l(x)$, there is no remote term $S_{\tau}$ for $20 \leq$ $\tau \leq 2^{34}$ for which the size of set $I$ is less than 5 . Thus, the WG-8 stream cipher is also resistant to this general distinguishing attack.

\subsection{Discrete Fourier Transform Attack}

The Discrete Fourier Transform (DFT) attack is a new type of attack to recover the internal state of a filtering generator, which was first proposed by Rønjom and Helleseth in [36] and extended to attacking filtering generators over $\mathbb{F}_{2^{n}}$ by Gong et al. in [20]. For mounting the DFT attack against the WG-8 stream cipher, an attacker needs to obtain $2^{33.32}$ (i.e., the linear complexity) consecutive keystream bits. Hence, the online complexity of this attack for recovering the internal state is $2^{33.32}$, after an offline computation with complexity $2^{48.49}$. For typical lightweight embedded applications like RFID systems, a reader and a tag only exchange 32-bit random numbers in each communication session. Hence, an attacker can never obtain $2^{33.32}$ consecutive keystream bits.

\subsection{Time-Memory-Data Tradeoff Attack}

The Time-Memory-Data (TMD) tradeoff attack [4] is a generic cryptanalytic attack that is applicable to any stream cipher, especially those with low sampling resistance. The complexity of the TMD tradeoff attack is $O\left(2^{\frac{n}{2}}\right)$, where $n$ is the size of the internal state. For the WG-8 stream cipher, the size of the internal state is 160 bit and thus the complexity of launching a TMD attack is at least $2^{80}$. Moreover, the sampling resistance of the WG-8 stream cipher is high due to the usage of the WGT$8\left(x^{19}\right)$ as the filtering function. The ANF representation of the WGT-8 $\left(x^{19}\right)$ contains 109 terms, among which only four terms are linear and other terms have degree greater than 2 and less than 8 . Hence, only by fixing 7 out of 8 variables can one obtain a linear equation.

\subsection{Related-key Attack}

A related-key attack is a cryptanalytic attack that can also be applied to a stream cipher and allows to launch a key-recovery attack. In [12], Ding et al. proposed a key-recovery attack in the related key settings on WG-8 stream cipher by exploiting the slide property of WG8 stream cipher. The key-recovery attack has a time complexity of $2^{53.32}$ and needs $2^{52}$ chosen IVs. From the DFT attack on WG-8 stream cipher, it can be observed that the DFT attack has an online time complexity of $2^{33.32}$ and data complexity of $2^{33.32}$, which are much lower than the complexities of Ding et al.'s key-recovery attack. We believe that the key-recovery attack by Ding et al. is not a real threat on our WG-8 stream cipher.

\subsection{Mihaljević et al.'s Attack}

In [30], Mihaljević et al. proposed an internal state recovery attack on Grain-v1 by exploiting the normality of the filtering function of Grain-v1. Their cryptanalysis exploits three facts: a) the bias towards a subset of the internal states; b) recovering some partial key bits; and c) adaptation of the TMD tradeoff approach to use the bias of the internal state. We note that the bias of the internal states is detected by using the normality of the filtering function. Since an LFSR that generates an $m$-sequence is used to update the internal state of WG-8 stream cipher, no such bias on the internal state exists in the internal state. Moreover, the normality of WGT-8 $\left(x^{19}\right)$ is 3. Therefore, Mihaljević et al.'s attack cannot be applied to the WG-8 stream cipher.

\section{Efficient Software Implementation of the Stream Cipher WG-8}

In this section, we address efficient software implementation of the WG-8 cipher on low-power microcontrollers. For each platform we provide three implementation variants that deal with trade-offs among speed, code size, and energy consumption.

\subsection{Implementation of the WG-8 Permutation Module WGP-8 $\left(x^{19}\right)$}

The most complicated WGP-8 $\left(x^{19}\right)$ module can be implemented using three different methods: a) a 256-byte direct look-up table; b) a 34-byte coset leader based lookup table; or c) tower field (TF) arithmetic.

Directly Look-up Table (DLT) Approach. Depending on the bases used, one can precompute the WG-8 
permutation with decimation $d=19$ by

$$
\text { WGP-8 }\left(x^{19}\right)=q\left(x^{19}+1\right)+1
$$

for all elements $x \in \mathbb{F}_{2^{8}}$. Hence, a 256-byte look-up table $T_{\text {WGP-8 }}$ can be generated to compute WGP-8 $\left(x^{19}\right)$.

Coset Leader Based Look-up Table (CLT) Approach. This approach assumes that a normal basis is used to represent elements in $\mathbb{F}_{2^{8}}$ and uses the essential property of the WG-8 permutation with decimation $d$ below:

$$
\begin{aligned}
& \text { WGP-8 }\left(\left(x^{2^{i}}\right)^{d}\right)=q\left(\left(x^{2^{i}}\right)^{d}+1\right)+1 \\
& =q\left(\left(x^{d}\right)^{2^{i}}+1\right)+1=\left(q\left(x^{d}+1\right)\right)^{2^{i}}+1 \\
& =\left(q\left(x^{d}+1\right)+1\right)^{2^{i}}=\left(\text { WGP-8 }\left(x^{d}\right)\right)^{2^{i}}
\end{aligned}
$$

for $\quad x \in \mathbb{F}_{2^{8}}$ and $i=0,1, \ldots, 7$. According to the Equation (1), if we know the WG-8 permutation WGP-8 $\left(x^{d}\right)$ for an element $x \in \mathbb{F}_{2^{8}}$, we can easily obtain the WG-8 permutation WGP-8 $\left(\left(x^{2^{i}}\right)^{d}\right)$ for the entire conset $\left\{x^{2}, x^{2^{2}}, \ldots, x^{2^{7}}\right\}$ of $x$ by cyclically shifting WGP-8 $\left(x^{d}\right)$ to the right by $i$ positions, provided that a normal basis is employed to represent finite field elements. The complete cosets and coset leaders of $\mathbb{F}_{2^{8}}$ (in hexadecimal notation) are shown in Table 1. We note that under the normal basis representation the elements in $\mathbb{F}_{2^{8}}$ have been grouped into 34 different cosets except for 0 and 1. Since WGP-8(0) $=0 \times 00$ and WGP-8(1) $=$ 0xFF, we only need to generate a 34-byte look-up table $T_{\text {Co-WGP-8 }}$ for storing the WG-8 permutation results for each coset leader. Here we present the following Algorithm 1 that uses the table $T_{\text {Co-WGP-8 }}$ to compute WGP-8 $\left(x^{d}\right)$ for any $x \in \mathbb{F}_{2^{8}}$.

Tower Field Arithmetic (TFA) Based Approach. The software implementation of the WGP-8 $\left(x^{19}\right)$ module involves the arithmetic (i.e., addition, multiplication, and exponentiation) over finite field $\mathbb{F}_{2^{8}}$. Although we can directly implement all the operations over $\mathbb{F}_{2^{8}}$, it is well known that using the isomorphic tower constructions of $\mathbb{F}_{2^{8}}$ might save the memory consumption. Therefore, we investigate the tower construction $\mathbb{F}_{\left(2^{4}\right)^{2}}$ in this work.

Tower Construction $\mathbb{F}_{\left(2^{4}\right)^{2}}$ and Its Arithmetic. To obtain the tower construction $\mathbb{F}_{\left(2^{4}\right)^{2}}$, we first construct $\mathbb{F}_{2^{4}}$ by using an irreducible polynomial $e(X)$

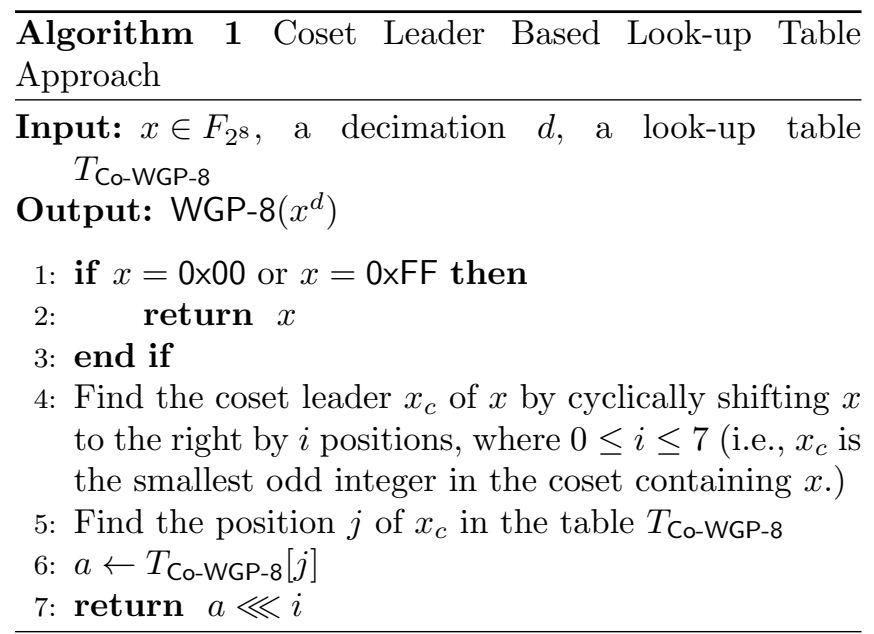

of degree 4 over $\mathbb{F}_{2}$, and then construct $\mathbb{F}_{\left(2^{4}\right)^{2}}$ by using a certain irreducible polynomial $f(X)$ of degree 2 over $\mathbb{F}_{2^{4}}$. In our tower construction, we use $e(X)=X^{4}+$ $X^{3}+1$ with its polynomial basis $\left\{1, \alpha, \alpha^{2}, \alpha^{3}\right\}$ for $\mathbb{F}_{2^{4}}$ and $f(X)=X^{2}+X+\alpha$ with its normal basis $\left\{\beta, \beta^{16}\right\}$ for $\mathbb{F}_{\left(2^{4}\right)^{2}}$, where $\alpha=\omega^{119} \in \mathbb{F}_{2^{4}}$ and $\beta=\omega^{7} \in \mathbb{F}_{\left(2^{4}\right)^{2}}$ are zeros of the polynomials $e(X)$ and $f(X)$, respectively.

Arithmetic operations in $\mathbb{F}_{2^{4}}$. The arithmetic in $\mathbb{F}_{2^{4}}$ is conducted with the aid of a $4 \times 4$ exponentiation table $T_{\text {exp }}$ and a $4 \times 4$ logarithm table $T_{l o g}$. While the table $T_{\text {exp }}$ stores exponentiation $\alpha^{i}, i=0,1, \ldots, 14$, the table $T_{l o g}$ keeps the exponent $i$ for each $\alpha^{i}, i=0,1, \ldots, 14$. Let $A=a_{0}+a_{1} \alpha+a_{2} \alpha^{2}+a_{3} \alpha^{3}$ and $B=b_{0}+b_{1} \alpha+$ $b_{2} \alpha^{2}+b_{3} \alpha^{3}$ be two non-zero elements in $\mathbb{F}_{2^{4}}$, where $a_{i}, b_{i} \in \mathbb{F}_{2}, i=0,1,2,3$. We can perform the arithmetic in $\mathbb{F}_{2^{4}}$ as follows:

$$
\begin{gathered}
A B=T_{\text {exp }}\left[\left(T_{l o g}\left[\left(a_{0}, a_{1}, a_{2}, a_{3}\right)\right]+\right.\right. \\
\left.\left.T_{l o g}\left[\left(b_{0}, b_{1}, b_{2}, b_{3}\right)\right]\right) \bmod 15\right], \\
A^{2}=T_{\text {exp }}\left[\left(T_{l o g}\left[\left(a_{0}, a_{1}, a_{2}, a_{3}\right)\right] \ll 1\right) \bmod 15\right], \\
\alpha A=T_{\text {exp }}\left[\left(T_{l o g}\left[\left(a_{0}, a_{1}, a_{2}, a_{3}\right)\right]+1\right) \bmod 15\right] .
\end{gathered}
$$

Arithmetic operations in $\mathbb{F}_{\left(2^{4}\right)^{2}}$. Let $A=a_{0} \beta+$ $a_{1} \beta^{16}$ and $B=b_{0} \beta+b_{1} \beta^{16}$, where $a_{0}, a_{1}, b_{0}, b_{1} \in \mathbb{F}_{2^{4}}$. A multiplication $A B$ in $\mathbb{F}_{\left(2^{4}\right)^{2}}$ is computed as follows:

$$
\begin{aligned}
A B & =\left(a_{0} \beta+a_{1} \beta^{16}\right)\left(b_{0} \beta+b_{1} \beta^{16}\right) \\
& =\left(c \alpha \oplus a_{0} b_{0}\right) \beta+\left(c \alpha \oplus a_{1} b_{1}\right) \beta^{16}
\end{aligned}
$$


Table 1. The Cosets and Coset Leaders of $\mathbb{F}_{2^{8}}$

\begin{tabular}{|c|c|c|c|c|c|c|c|c|c|c|c|c|c|c|c|}
\hline Coset Leader & \multicolumn{7}{|c|}{ Coset } & Coset Leader & \multicolumn{7}{|c|}{ Coset } \\
\hline $0 \times 00$ & - & - & - & - & - & - & - & $0 \times 27$ & $0 \times 4 \mathrm{E}$ & $0 \times 9 C$ & $0 \times 39$ & $0 \times 72$ & $0 \times E 4$ & $0 \times \mathrm{C} 9$ & $0 \times 93$ \\
\hline $0 \times 01$ & $0 \times 02$ & $0 \times 04$ & $0 \times 08$ & $0 \times 10$ & $0 \times 20$ & $0 \times 40$ & $0 \times 40$ & $0 \times 2 B$ & $0 \times 56$ & $0 \times A C$ & $0 \times 59$ & $0 \times B 2$ & $0 \times 65$ & $0 \times C A$ & $0 \times 95$ \\
\hline $0 \times 03$ & $0 \times 06$ & $0 \times 0 \mathrm{C}$ & $0 \times 18$ & $0 \times 30$ & $0 \times 60$ & $0 \times C 0$ & $0 \times 81$ & $0 \times 2 D$ & $0 \times 5 \mathrm{~A}$ & 0xB4 & $0 \times 69$ & $0 \times \mathrm{D} 2$ & $0 \times A 5$ & $0 \times 4 B$ & $0 \times 96$ \\
\hline $0 \times 05$ & $0 \times 0 \mathrm{~A}$ & $0 \times 14$ & $0 \times 28$ & $0 \times 50$ & $0 \times A 0$ & $0 \times 41$ & $0 \times 82$ & $0 \times 2 F$ & $0 \times 5 E$ & $0 \times B C$ & $0 \times 79$ & $0 \times \mathrm{F} 2$ & $0 \times E 5$ & $0 \times \mathrm{CB}$ & $0 \times 97$ \\
\hline $0 \times 07$ & $0 \times 0 \mathrm{E}$ & $0 \times 1 C$ & $0 \times 38$ & $0 \times 70$ & $0 \times E 0$ & $0 \times \mathrm{C} 1$ & $0 \times 83$ & $0 \times 33$ & $0 \times 66$ & $0 \times \mathrm{CCC}$ & $0 \times 99$ & - & - & - & - \\
\hline $0 \times 09$ & $0 \times 12$ & $0 \times 24$ & $0 \times 48$ & $0 \times 90$ & $0 \times 21$ & $0 \times 42$ & $0 \times 84$ & $0 \times 35$ & $0 \times 6 \mathrm{~A}$ & 0xD4 & 0xA9 & $0 \times 53$ & $0 \times A 6$ & $0 \times 4 D$ & $0 \times 9 \mathrm{~A}$ \\
\hline $0 \times 0 B$ & $0 \times 16$ & $0 \times 2 C$ & $0 \times 58$ & $0 \times B 0$ & $0 \times 61$ & $0 \times C 2$ & $0 \times 85$ & $0 \times 37$ & $0 \times 6 \mathrm{E}$ & $0 \times \mathrm{DC}$ & 0xB9 & $0 \times 73$ & 0xE6 & $0 \times C D$ & $0 \times 9 B$ \\
\hline $0 \times 0 \mathrm{D}$ & $0 \times 1 \mathrm{~A}$ & $0 \times 34$ & $0 \times 68$ & $0 \times \mathrm{DD} 0$ & $0 \times A 1$ & $0 \times 43$ & $0 \times 86$ & $0 \times 3 B$ & $0 \times 76$ & 0xEC & 0xD9 & $0 \times B 3$ & $0 \times 67$ & $0 \times \mathrm{CE}$ & $0 \times 9 D$ \\
\hline $0 \times 0 \mathrm{~F}$ & $0 \times 1 \mathrm{E}$ & $0 \times 3 C$ & $0 \times 78$ & $0 \times F 0$ & 0xE1 & $0 \times C 3$ & $0 \times 87$ & $0 \times 3 D$ & $0 \times 74$ & $0 \times F 4$ & 0xE9 & $0 \times \mathrm{D} 3$ & $0 \times A 7$ & $0 \times 4 \mathrm{~F}$ & $0 \times 9 E$ \\
\hline $0 \times 11$ & $0 \times 22$ & $0 \times 44$ & $0 \times 88$ & - & - & - & - & $0 \times 3 F$ & $0 \times 7 \mathrm{E}$ & $0 \times \mathrm{xFC}$ & 0xF9 & $0 \times \mathrm{F} 3$ & 0xE7 & $0 \times C F$ & $0 \times 9 \mathrm{~F}$ \\
\hline $0 \times 13$ & $0 \times 26$ & $0 \times 4 C$ & $0 \times 98$ & $0 \times 31$ & $0 \times 62$ & $0 \times C 4$ & $0 \times 89$ & $0 \times 55$ & $0 \times A A$ & - & - & - & - & - & - \\
\hline $0 \times 15$ & $0 \times 2 \mathrm{~A}$ & $0 \times 54$ & $0 \times A 8$ & $0 \times 51$ & $0 \times A 2$ & $0 \times 45$ & $0 \times 8 \mathrm{~A}$ & $0 \times 57$ & $0 \times A E$ & $0 \times 5 \mathrm{D}$ & $0 \times B A$ & $0 \times 75$ & $0 \times E A$ & 0xD5 & $0 \times A B$ \\
\hline $0 \times 17$ & $0 \times 2 \mathrm{E}$ & $0 \times 5 \mathrm{C}$ & $0 \times B 8$ & $0 \times 71$ & $0 \times E 2$ & $0 \times C 5$ & $0 \times 8 B$ & $0 \times 5 B$ & $0 \times B 6$ & $0 \times 6 \mathrm{D}$ & $0 \times \mathrm{DA}$ & $0 \times B 5$ & $0 \times 6 B$ & 0xD6 & $0 \times A D$ \\
\hline $0 \times 19$ & $0 \times 23$ & $0 \times 64$ & $0 \times C 8$ & $0 \times 91$ & $0 \times 23$ & $0 \times 46$ & $0 \times 8 \mathrm{C}$ & $0 \times 5 F$ & $0 \times B E$ & $0 \times 7 D$ & 0xFA & $0 \times F 5$ & $0 \times E B$ & 0xD7 & $0 \times A F$ \\
\hline $0 \times 1 B$ & $0 \times 36$ & $0 \times 6 C$ & 0xD8 & $0 \times B 1$ & $0 \times 63$ & $0 \times \mathrm{C} 6$ & $0 \times 8 D$ & $0 \times 6 \mathrm{~F}$ & $0 \times \mathrm{DE}$ & $0 \times B D$ & $0 \times 7 B$ & $0 \times F 6$ & $0 \times E D$ & $0 \times \mathrm{DB}$ & $0 \times B 7$ \\
\hline $0 \times 1 \mathrm{D}$ & $0 \times 3 \mathrm{~A}$ & $0 \times 74$ & 0xE8 & 0xD1 & $0 \times A 3$ & $0 \times 47$ & $0 \times 8 \mathrm{E}$ & $0 \times 77$ & 0xEE & $0 \times \mathrm{DD}$ & $0 \times B B$ & - & - & - & - \\
\hline $0 \times 1 F$ & $0 \times 3 E$ & $0 \times 7 C$ & 0xF8 & $0 \times \mathrm{F} 1$ & $0 \times E 3$ & $0 \times \mathrm{C} 7$ & $0 \times 8 \mathrm{~F}$ & $0 \times 7 F$ & $0 \times \mathrm{FE}$ & 0xFD & 0xFB & $0 \times \mathrm{FF} 7$ & $0 \times E F$ & 0xDF & $0 \times B F$ \\
\hline $0 \times 25$ & $0 \times 4 \mathrm{~A}$ & $0 \times 94$ & $0 \times 29$ & $0 \times 52$ & $0 \times A 4$ & $0 \times 49$ & $0 \times 92$ & $0 \times F F$ & - & - & - & - & - & - & - \\
\hline
\end{tabular}

where $c=\left(a_{0} \oplus a_{1}\right)\left(b_{0} \oplus b_{1}\right)$. For a non-zero element $A \in$ $\mathbb{F}_{\left(2^{4}\right)^{2}}$, the squaring of $A$ is calculated as follows:

$$
\begin{aligned}
A^{2} & =\left(a_{0} \beta+a_{1} \beta^{16}\right)^{2} \\
& =\left[\left(a_{0} \oplus a_{1}\right)^{2} \alpha \oplus a_{0}^{2}\right] \beta+\left[\left(a_{0} \oplus a_{1}\right)^{2} \alpha \oplus a_{1}^{2}\right] \beta^{16} .
\end{aligned}
$$

The Frobenius mapping of $A$ with respect to $\mathbb{F}_{2^{4}}$, which is the $16^{\text {th }}$ power operation, is computed as follows:

$A^{2^{4}}=\left(a_{0} \beta+a_{1} \beta^{16}\right)^{16}=a_{0} \beta^{16}+a_{1} \beta^{256}=a_{1} \beta+a_{0} \beta^{16}$.

Implementation of WGP-8 $\left(x^{19}\right)$ Module. For an element $x \in \mathbb{F}_{2^{8}}$, the WGP-8 $\left(x^{19}\right)$ can be computed as follows:

$$
\begin{aligned}
& \text { WGP-8 }\left(x^{19}\right)=q\left(x^{19}+1\right)+1 \\
& =y+y^{2^{3}+1}+y^{2^{6}}\left(y^{2^{3}+1}+y^{2^{3}-1}\right)+y^{2^{3}\left(2^{3}-1\right)+1}+1,
\end{aligned}
$$

where $y=x^{19}+1=x^{2^{4}} \cdot x^{2} \cdot x+1$. Note that for the tower construction $\mathbb{F}_{\left(2^{4}\right)^{2}}, 1$ can be denoted by the vector $(1,0,0,0,1,0,0,0)$. Therefore, the addition with 1 under the TF representation is equivalent to XORing with a constant 0x88.

\subsection{Implementation of the Trace Computation} Module $\operatorname{Tr}(\cdot)$

Depending on the bases chosen, the trace of an element $x \in \mathbb{F}_{2^{8}}$ can be computed as shown in Table 2.

\subsection{Implementation of the Multiplication by $\omega$ Module}

The multiplication by $\omega$ module can be implemented using either finite field arithmetic or an $8 \times 8$ look-up table.

Multiplication by $\omega$ Using Finite Field Arithmetic. We consider the following three cases when the $\mathrm{PB}, \mathrm{NB}$, and TF are used to represent finite field elements, respectively. With the PB representation, the multiplication of an element $x \in \mathbb{F}_{2^{8}}$ by $\omega$ can be computed as follows:

$$
\begin{aligned}
x \cdot \omega & =x_{0} \omega+x_{1} \omega^{2}+\cdots+x_{6} \omega^{7}+x_{7} \omega^{8} \\
& =x_{7}+x_{0} \omega+\left(x_{1} \oplus x_{7}\right) \omega^{2}+\left(x_{2} \oplus x_{7}\right) \omega^{3}+ \\
& \left(x_{3} \oplus x_{7}\right) \omega^{4}+x_{4} \omega^{5}+x_{5} \omega^{6}+x_{6} \omega^{7} .
\end{aligned}
$$

Therefore, the result of $x \cdot \omega$ is represented as an 8-bit vector $\left(x_{7}, x_{0}, x_{1} \oplus x_{7}, x_{2} \oplus x_{7}, x_{3} \oplus x_{7}, x_{4}, x_{5}, x_{6}\right)$ with respect the $\mathrm{PB}$. 
Table 2. Trace Computation of an Element $x \in \mathbb{F}_{2^{8}}$ Using Different Bases

\begin{tabular}{|c|c|c|}
\hline Basis & Element Representation & $\operatorname{Tr}(x)$ \\
\hline \hline Polynomial Basis (PB) & $x_{0}+x_{1} \omega+\cdots+x_{7} \omega^{7}$ & $x_{5}$ \\
\hline Normal Basis (NB) & $x_{0} \theta+x_{1} \theta^{2}+\cdots+x_{7} \theta^{2^{7}}$ & $\bigoplus_{i=0}^{7} x_{i}$ \\
\hline Tower Field (TF) & $\begin{array}{c}\left(x_{0}+x_{1} \alpha+x_{2} \alpha^{2}+x_{3} \alpha^{3}\right) \beta+ \\
\left(x_{4}+x_{5} \alpha+x_{6} \alpha^{2}+x_{7} \alpha^{3}\right) \beta^{16}\end{array}$ & $x_{1} \oplus x_{2} \oplus x_{3} \oplus x_{5} \oplus x_{6} \oplus x_{7}$ \\
\hline
\end{tabular}

With the NB representation, the multiplication of an element $x \in \mathbb{F}_{2^{8}}$ by $\omega$ can be calculated as follows:

$$
\begin{aligned}
x \cdot \omega & =\left(x_{0} \theta+x_{1} \theta^{2}+\cdots+x_{6} \theta^{2^{6}}+x_{7} \theta^{2^{7}}\right) \cdot \omega \\
& =\mathbf{M} \cdot\left(x_{0}, x_{1}, \cdots, x_{6}, x_{7}\right)^{T},
\end{aligned}
$$

where the matrix $\mathbf{M}$ is given below:

$$
\mathbf{M}=\left(\begin{array}{llllllll}
1 & 1 & 1 & 0 & 1 & 0 & 1 & 1 \\
0 & 0 & 0 & 0 & 1 & 1 & 1 & 0 \\
1 & 0 & 1 & 0 & 1 & 0 & 0 & 1 \\
1 & 0 & 1 & 1 & 1 & 0 & 0 & 0 \\
0 & 0 & 1 & 0 & 1 & 1 & 1 & 0 \\
0 & 1 & 1 & 0 & 0 & 1 & 1 & 1 \\
1 & 0 & 1 & 1 & 1 & 1 & 0 & 0 \\
0 & 1 & 1 & 0 & 1 & 0 & 1 & 1
\end{array}\right) .
$$

With the TF representation, the multiplication of an element $x \in \mathbb{F}_{2^{8}}$ by $\omega$ can be calculated as follows:

$$
\begin{aligned}
x \cdot \omega= & {\left[\left(x_{0}+x_{1} \alpha+x_{2} \alpha^{2}+x_{3} \alpha^{3}\right) \beta+\right.} \\
& \left.\left(x_{4}+x_{5} \alpha+x_{6} \alpha^{2}+x_{7} \alpha^{3}\right) \beta^{16}\right] \cdot \omega \\
= & \mathbf{M}^{\prime} \cdot\left(x_{0}, x_{1}, \cdots, x_{6}, x_{7}\right)^{T},
\end{aligned}
$$

where the matrix $\mathbf{M}^{\prime}$ is given below:

$$
\mathbf{M}^{\prime}=\left(\begin{array}{llllllll}
1 & 0 & 1 & 1 & 1 & 0 & 0 & 1 \\
0 & 1 & 0 & 1 & 1 & 1 & 0 & 0 \\
1 & 0 & 1 & 0 & 0 & 1 & 1 & 0 \\
0 & 1 & 1 & 0 & 0 & 0 & 1 & 0 \\
1 & 0 & 0 & 1 & 0 & 1 & 1 & 1 \\
1 & 1 & 0 & 0 & 0 & 0 & 1 & 1 \\
0 & 1 & 1 & 0 & 0 & 0 & 0 & 1 \\
0 & 0 & 1 & 0 & 1 & 1 & 1 & 1
\end{array}\right) .
$$

Multiplication by $\omega$ Using Look-Up Tables. Based on the Equations (2)-(4), one can generate 256-byte look-up tables with respect to the chosen bases.

\subsection{Implementation Platforms and Development Tools}

In this section, we briefly describe two low-power microcontrollers for implementing the WG-8 stream cipher as well as the corresponding development tools.

8-Bit Microcontroller ATmega128L and Development Tool. The low-power 8-bit microcontroller ATmega128L [1] from Atmel is based on the AVR enhanced RISC architecture with 128 Kbytes of InSystem Self-Programmable Flash, 4 Kbytes EEPROM and 8 Kbytes Internal SRAM. It is equipped with 133 highly-optimized instructions and most of them can be executed within one clock cycle. Moreover, the clock frequency of the ATmega128 $\mathrm{L}$ can run from 0 to $8 \mathrm{MHz}$ and the power supplies can go from 2.7 to $5.5 \mathrm{~V}$. We use the latest integrated development environment Atmel Studio 6.0 [2] from Atmel for implementing and testing the performance of the WG-8 on the target platform.

16-Bit Microcontroller MSP430F1611 and Development Tool. The 16-bit microcontroller MSP430F1611 [40] from Texas Instruments has a traditional vonNeumann architecture with 48 Kbytes Flash memory and 10 Kbytes RAM. All special function registers, peripherals, RAM and Flash/ROM share the same address space. The clock frequency of the MSP430F1611 ranges from 0 to $8 \mathrm{MHz}$ and the power supplies can go from 1.8 to $3.6 \mathrm{~V}$. The MSP430F1611 features 27 instructions and 7 different addressing modes that provide great flexibility in data manipulation. To implement and simulate the WG-8 on the target platform, we use the CrossWorks for MSP430 Version 2.1 from Rowley Associates [37]. 


\subsection{Experimental Results and Comparisons}

In this section, we report our experimental results for implementing the stream cipher WG-8 on the low-power microcontrollers ATmega128L and MSP430F1611 and compare our results with other lightweight-cryptography implementations on the same or similar platforms. We focus on three major performance criteria for implementing cryptographic primitives on resource-constrained environments, namely throughput, code size, and energy consumption (i.e., energy/bit). Table 3 compares our implementation results with previous work in terms of the aforementioned three performance criteria. Note that we estimate the per bit energy consumptions by the formula: energy $/$ bit $=\frac{\text { Supply Voltage } \times \text { Current } \times \text { Cycles }}{\text { Clock Frequency } \times \text { Number of Bits }}$, which is based on the typical current consumption of a low-power microcontroller for the given clock frequency and supply voltage.

From Table 3, we note that on 8-bit ATmega microcontrollers the throughput of WG- 8 is about $2 \sim 15$ times higher than that of stream ciphers Grain, Trivium, Salsa20, and WG-7, block ciphers PRESENT-80 and XTEA as well as the hybrid cipher Hummingbird, whereas the energy consumption of WG-8 is around $2 \sim 220$ times smaller than that of those ciphers. Moreover, WG8 has the comparable throughput and energy efficiency with the hybrid cipher Hummingbird-2 (optimized with assembly language). On the 8-bit platform, WG-8 is less efficient than $A E S$ in terms of throughput and energy consumption. The main reason is that WG-8 is a bitoriented stream cipher whereas AES is a block cipher with block size 128-bit. Furthermore, the code size of WG-8 is medium and the SRAM usage of WG-8 is small among all the lightweight implementations.

On 16-bit MSP430 microcontrollers, the throughput of WG-8 is about $1 \sim 20$ times higher than that of the stream cipher WG-7 as well as block ciphers PRINTcipher48, AES, PRESENT-80, and KLEIN-64, whereas the energy efficiency is comparable with that of those ciphers. While WG-8 has similar throughput and energy efficiency as the hybrid cipher Hummingbird, it is less efficient when compared to the Hummingbird-2 cipher. The main reason comes from the optimization with the assembly language in the speed-optimized Hummingbird-2 implementation. Furthermore, the code size of WG-8 is about $2 \sim 7$ times smaller than block ciphers PRINTcipher-48, AES, PRESENT-80, and KLEIN64 as well as the hybrid cipher Hummingbird-2, and is comparable with the Hummingbird cipher. Regarding to the SRAM usage, the stream cipher WG-8 is superior to other block cipher and stream ciphers.

In addition, for the three implementation variants, we note that on both 8-bit and 16-bit platforms the DLT method is consistently better than both CLT and TFA methods with respect to throughput and energy consumption. The reason lies in the efficient memory access for look-up tables on both microcontrollers.

\section{Efficient Hardware Implementation of the Stream Cipher WG-8}

Efficient hardware implementation of the WG-8 stream cipher on both FPGA and ASIC platforms has been extensively investigated in [42]. One look-up table based and three tower field based hardware architectures were proposed and compared to each other in terms of throughput, area, and power consumption. The experimental results show that a direct look-up table based hardware architecture can achieve a maximum throughput of $190 \mathrm{Mbps}$ (resp. $500 \mathrm{Mbps}$ ) and require 137 slices (resp. 1786 Gate Equivalents (GEs)) on a Xilinx Spartan-3 FPGA (resp. 65nm CMOS ASIC) platform, at the cost of the dynamic power consumption of $0.005 \mathrm{~W}$ (resp. $0.983 \mathrm{~mW}$ ). Moreover, the look-up table based method is optimal with respect to the defined performance metrics when compared to the tower field based approaches. For certain performance metrics, the WG-8 hardware core compares well with the lightweight stream ciphers Grain [22], Trivium [7], and MICKEY [3].

\section{Conclusion}

In this paper, we present a lightweight stream cipher WG8 targeted for resource-constrained devices like RFID tags, smart cards, and wireless sensor nodes, which inherits all the good randomness and cryptographic properties of the well-known WG stream cipher family. A detailed cryptanalysis shows that WG-8 is resistant to the most common attacks against stream ciphers. Moreover, the software implementations on low-power microcontrollers demonstrate the high performance and 
Table 3. Performance Comparison of Lightweight-Cryptography Implementations on Low-Power Microcontrollers

\begin{tabular}{|c|c|c|c|c|c|c|c|c|}
\hline \multirow[t]{2}{*}{$\begin{array}{c}\text { Low-Power } \\
\text { Microcontroller }\end{array}$} & \multirow[t]{2}{*}{$\begin{array}{l}\text { Cryptographic } \\
\text { Primitive }\end{array}$} & \multirow[t]{2}{*}{$\begin{array}{c}\text { Clock Freq. } \\
{[\mathrm{MHz}]}\end{array}$} & \multirow[t]{2}{*}{$\begin{array}{l}\text { Opt. Goal/ } \\
\text { Method }\end{array}$} & \multicolumn{2}{|c|}{$\begin{array}{c}\text { Memory Usage } \\
\text { [byte] }\end{array}$} & \multirow[t]{2}{*}{$\begin{array}{l}\text { Setup } \\
\text { [cycle] }\end{array}$} & \multirow[t]{2}{*}{$\begin{array}{l}\text { Throughput } \\
\text { [Kbits/sec] }\end{array}$} & \multirow[t]{2}{*}{$\begin{array}{c}\text { Energy/Bit } \\
{[\mathrm{nJ}]}\end{array}$} \\
\hline & & & & Flash & SRAM & & & \\
\hline \multirow{16}{*}{ ATmega } & \multirow{2}{*}{ AES [33] } & \multirow{16}{*}{$8 \mathrm{MHz}$} & RAM & 1,912 & 176 & 789 & 475.6 & 179 \\
\hline & & & Speed & 1,912 & 256 & 747 & 513.8 & 165 \\
\hline & \multirow{2}{*}{ PRESENT-80 [35] } & & Size & 1,474 & 32 & - & 0.99 & 85,819 \\
\hline & & & Speed & 2,398 & 528 & - & 66.7 & 1,274 \\
\hline & \multirow{2}{*}{ Hummingbird [16] } & & Size & 1,308 & - & 14,735 & 34.9 & 2,433 \\
\hline & & & Speed & 10,918 & - & 8,182 & 91.5 & 929 \\
\hline & \multirow{2}{*}{ Hummingbird-2 [17] } & & RAM & 3,600 & 114 & 2,970 & 171.8 & 495 \\
\hline & & & Speed & 3,200 & 1,500 & 1,800 & 258.6 & 329 \\
\hline & XTEA [34] & & Speed & 820 & - & - & 51.7 & 1,645 \\
\hline & Grain[34] & & Speed & 778 & 20 & 107,336 & 12.9 & 6,556 \\
\hline & Trivium[34] & & Speed & 424 & 36 & 775,726 & 12.0 & 7,066 \\
\hline & Salsa20[29] & & Speed & 3,842 & 258 & 318 & 83.7 & 101,564 \\
\hline & WG-7[27] & & Size & 938 & - & 20,917 & 34.0 & 2,497 \\
\hline & & & TFA & 2,450 & 20 & 99,702 & 3.58 & 23,739 \\
\hline & WG-8 & & CLT & 2,238 & 148 & 10,683 & 31.7 & 2,683 \\
\hline & & & DLT & 1,984 & 20 & 1,379 & 185.5 & 458 \\
\hline \multirow{12}{*}{ MSP430 } & PRINTcipher-48 [19] & \multirow{12}{*}{$8 \mathrm{MHz}$} & Speed & 6,424 & 48 & - & 4.5 & 153 \\
\hline & AES [19] & & Speed & 10,898 & 218 & - & 78.0 & 154 \\
\hline & PRESENT-80 [19] & & Speed & 6,424 & 288 & - & 19.4 & 619 \\
\hline & KLEIN-64 [19] & & Speed & 6,424 & 288 & - & 65.0 & 185 \\
\hline & \multirow{2}{*}{ Hummingbird [16] } & & Size & 1,064 & - & 9,667 & 53.0 & 226 \\
\hline & & & Speed & 1,360 & - & 4,824 & 104.9 & 114 \\
\hline & \multirow{2}{*}{ Hummingbird-2 [17] } & & Size & 770 & 50 & 5,984 & 84.2 & 143 \\
\hline & & & Speed & 3,648 & 114 & 1,361 & 356.5 & 34 \\
\hline & WG-7[27] & & Size & 1,050 & - & 18,379 & 21.0 & 572 \\
\hline & \multirow{3}{*}{ WG-8 } & & TFA & 2,110 & 20 & 127,944 & 2.44 & 4,926 \\
\hline & & & CLT & 2,628 & 148 & 15,265 & 10.8 & 1,107 \\
\hline & & & DLT & 1,558 & 20 & 3,604 & 95.9 & 125 \\
\hline
\end{tabular}

low energy consumption of the WG-8 stream cipher, when compared to most of previous block ciphers and stream ciphers. Therefore, the stream cipher WG-8 is a competitive candidate for securing a wide range of smart devices and embedded applications.

\section{Ackowledgement}

The authors would like to thank the reviewers for their helpful and constructive comments that greatly improve the final version of this paper. This work is supported by NSERC Discovery and ORF-RE grants.

\section{References}

[1] Atmel Corporation (2011) ATmega128(L): 8-bit Atmel Microcontroller with 128 KBytes In-System Programmable Flash, Available at http://www . atmel.com/ Images/doc2467.pdf.

[2] Atmel Corporation (2012) Atmel Studio 6 - The Integrated Development Environment, Available at http://www . atmel . com/microsite/atmel_studio6/.

[3] Babbage S. and Dodd M. (2006) The Stream Cipher MICKEY 2.0, ECRYPT Stream Cipher, Available at http://www.ecrypt.eu.org/stream/p3ciphers/ mickey/mickey_p3.pdf.

[4] Biryukov A. and Shamir A. (2000) Cryptanalytic Time/Memory/Data Tradeoffs for Stream Ciphers, 
Advances in Cryptology - ASIACRYPT 2000, LNCS 1976, T. Okamoto (Ed.), Berlin, Germany: SpringerVerlag, pp. 1-13.

[5] Bogdanov A. and Knudsen L. R. and Leander G. and Paar C. and Poschmann A. and Robshaw M. J. B. and Seurin Y. and Vikkelsoe C. (2007) PRESENT: An Ultra-Lightweight Block Cipher, The 9th International Workshop on Cryptographic Hardware and Embedded Systems - CHES 2007, LNCS 4727, P. Paillier and I. Verbauwhede (eds.), Berlin, Germany: Springer-Verlag, pp. 450-466.

[6] De Cannière C. and Dunkelman O. and Knežević M. (2009) KATAN and KTANTAN - A Family of Small and Efficient Hardware-Oriented Block Ciphers, The 11th International Workshop on Cryptographic Hardware and Embedded Systems - CHES 2009, LNCS 5747, C. Clavier and K. Gaj (eds.), Berlin, Germany: Springer-Verlag, pp. 272-288.

[7] De Cannière C. and Preneel B. (2005) Trivium - A Stream Cipher Construction Inspired by Block Cipher Design Principles, ECRYPT Stream Cipher, Available at http://www.ecrypt.eu.org/stream/papersdir/ 2006/021.pdf.

[8] Chen L. and Gong G. (2012) Communication System Security, Boca Raton, Florida, USA: Chapman \& Hall/CRC.

[9] Chepyzhov V.V. and Johansson T. and Smeets B.J.M. (2000) A Simple Algorithm for Fast Correlation Attacks on Stream Ciphers, The 7th International Workshop on Fast Software Encryption - FSE 2000, LNCS 1978, B. Schneier (Ed.), Berlin, Germany: Springer-Verlag, pp. 181-195.

[10] Courtois N. (2003) Fast Algebraic Attacks on Stream Ciphers with Linear Feedback, Advances in Cryptology - CRYPTO 2003, LNCS 2729, D. Boneh (Ed.), Berlin, Germany: Springer-Verlag, pp. 176-194.

[11] Courtois N. and Meier W. (2003) Algebraic Attacks on Stream Ciphers with Linear Feedback, Advances in Cryptology - EUROCRYPT 2003, LNCS 2656, E. Biham (Ed.), Berlin, Germany: Springer-Verlag, pp. 345-359.

[12] Ding L. and Jin C. and Guan, J. and Wang Q. (2014) Cryptanalysis of Lightweight WG-8 Stream Cipher, IEEE Transactions on Information Forensics and Security, vol.9, no.4, pp. $645-652$.

[13] Dinur I. and Shamir A. (2009) Cube Attacks on Tweakable Black Box Polynomials, Advances in Cryptology - EUROCRYPT'09, LNCS 5479, A. Joux (Ed.), Berlin, Germany: Springer-Verlag, pp. 278-299.
[14] Driessen B. and Hund R. and Willems C. and Paar C. and Holz T. (2012) Don't Trust Satellite Phones: A Security Analysis of Two Satphone Standards, The 33th IEEE Symposium on Security and Privacy - SE3P 2012, pp. 128-142.

[15] Eisenbarth T. and Kumar S. and Paar C. and Poschmann A. and Uhsadel L. (2007) A Survey of Lightweight-Cryptography Implementations, IEEE Design \& Test of Computers, vol. 24, no. 6, pp. 522-533.

[16] Engels D. and Fan X. and Gong G. and $\mathrm{Hu} \mathrm{H.}$ and Smith E.M. (2010) Hummingbird: Ultra-Lightweight Cryptography for Resource- Constrained Devices, FC 2010 Workshops, RLCPS, WECSR, and WLC 2010, LNCS 6054, R. Sion et al. (Eds.), Berlin, Germany: Springer-Verlag, pp. 3-18.

[17] Engels D. and Saarinen M.-J. O. and Schweitzer P. and Smith E.M. (2011) The Hummingbird-2 Lightweight Authenticated Encryption Algorithm, The 7th International Workshop on RFID Security and Privacy - RFIDSec 2011, LNCS 7055, A. Juels and C. Paar (Eds.), Berlin, Germany: Springer-Verlag, pp. 1931.

[18] Feldhofer M. and Wolkerstorfer J. and Rijmen V. (2005) AES Implementation on a Grain of Sand, IEE Proceedings Information Security, vol. 15, no. 1, pp. 1320.

[19] Gong Z. and Nikova S. and Law Y. (2012) KLEIN: A New Family of Lightweight Block Ciphers, The 7th International Workshop on RFID Security and Privacy RFIDSec 2011, LNCS 7055, A. Juels and C. Paar (Eds.), Berlin, Germany: Springer-Verlag, pp. 1-18.

[20] Gong G. and Rønjom S. and Helleseth T. and Hu H. (2011) Fast Discrete Fourier Spectra Attacks on Stream Ciphers, IEEE Transactions on Information Theory, vol. 57, No. 8, pp. 5555-5565.

[21] Guo J. and Peyrin T. and Poschmann A. and Robshaw M.J.B. (2011) The LED Block Cipher, The 13th International Workshop on Cryptographic Hardware and Embedded Systems - CHES 2011, LNCS 6917, B. Preneel and T. Takagi (eds.), Berlin, Germany: Springer-Verlag, pp. 326-341.

[22] Hell M. and Johansson T. and Meier W. (2007) Grain: A Stream Cipher for Constrained Environments, International Journal of Wireless and Mobile Computing, vol. 2, no. 1, pp. 86-93.

[23] Kaps J.-P. (2008) Chai-Tea, Cryptographic Hardware Implemenations of xTEA, The 9th International Conference on Cryptology in India - INDOCRYPT 2008, LNCS 5356, D. R. Chowdhury, V. Rijmen, and A. Das 
(eds.), Berlin, Germany: Springer-Verlag, pp. 363-375.

[24] Knudsen L. and Leander G. and Poschmann A. and Robshaw M. J. B. (2010) PRINTcipher: A Block Cipher for IC-Printing, The 12th International Workshop on Cryptographic Hardware and Embedded Systems - CHES 2010, LNCS 6225, S. Mangard and F.-X. Standaert (eds.), Berlin, Germany: Springer-Verlag, pp. 16-32.

[25] Leander G. and Paar C. and Poschmann A. and Schramm K. (2007) New Lightweight DES Variants, The 14th Annual Fast Software Encryption Workshop - FSE 200\%, LNCS 4593, A. Biryukov (ed.), Berlin, Germany: Springer-Verlag, pp. 196-210.

[26] Liu D. and Yang Y. and Wang J. and Min H. (2009) A Mutual Authentication Protocol for RFID Using IDEA, Auto-ID Labs White Paper, WP-HARDWARE-048, available at http://www.autoidlabs.org/uploads/ media/AUTOIDLABS-WP-HARDWARE-048.pdf.

[27] Luo Y. and Chai Q. and Gong G. and Lai X. (2010) WG-7: A Lightweight Stream Cipher with Good Cryptographic Properties, IEEE Global Communications Conference - GLOBECOM 2010, pp. 1-6.

[28] Meier W. and Staffelbach O. (1989) Fast Correlation Attacks on Certain Stream Ciphers, Journal of Cryptology, vol. 1, No. 3, pp. 159-176.

[29] Meiser G. and Eisenbarth T. and Lemke-Rust K. and Paar C. (2008) Efficient Implementation of eSTREAM Ciphers on 8-bit AVR Microcontrollers, International Symposium on Industrial Embedded Systems - SIES 2008, pp. 58-66.

[30] Mihaljević M.J. and Gangopadhyay S. and Paul G. and Imai H. (2012) Internal State Recovery of Grain-v1 Employing Normality Order of the Filter Function, IET Information Security, vol.6, No.2, pp.55-64.

[31] Nawaz Y. and Gong G. (2008) WG: A Family of Stream Ciphers with Designed Randomness Properties, Information Science, vol. 178, no. 7, pp. 1903-1916.

[32] Orumiehchiha M.A. and Pieprzyk J. and Steinfeld R. (2012) Cryptanalysis of WG-7: A Lightweight Stream Cipher, Cryptography and Communications, vol. 4, Iss. 3-4, pp. 277-285.

[33] Osvik D. A. and Bos J. W. and Stefan D. and Canright D. (2010) Fast Software AES Encryption, The 17th
International Workshop on Fast Software Encryption FSE 2010, LNCS 6147, S. Hong and T. Iwata (eds.), Berlin, Germany: Springer-Verlag, pp. 75-93.

[34] Otte D. (2012) AVR-Crypto-Lib, Available at http: //www.das-labor.org/wiki/AVR-Crypto-Lib/en.

[35] Poschmann A. (2009) Lightweight Cryptography Cryptographic Engineering for a Pervasive World, Ph.D. Thesis, Department of Electrical Engineering and Information Science, Ruhr-Universitäet Bochum, Bochum, Germany.

[36] Rønjom S. and Helleseth T. (2007) A New Attack on the Filtering Generator, IEEE Transactions on Information Theory, vol 53, No. 5, pp. 1752-1758.

[37] Rowley Associates, (2012) CrossWorks for MSP430, Available at http://www.rowley.co.uk/msp430/.

[38] Shibutani K. and Isobe T. and Hiwatari H. and Mitsuda A. and Akishita T. and Shirai T. (2011) Piccolo: An Ultra-Lightweight Blockcipher, The 13th International Workshop on Cryptographic Hardware and Embedded Systems - CHES 2011, LNCS 6917, B. Preneel and T. Takagi (eds.), Berlin, Germany: Springer-Verlag, pp. 342-357.

[39] Siegenthaler T. (1985) Decrypting a Class of Stream Ciphers Using Ciphertext Only, IEEE Transactions on Computers, vol. 34, No. 1, pp. 81-85.

[40] Texas Instuments Inc., MSP430F15x, MSP430F16x, MSP430F161x Mixed Signal Microcontroller, Available at http://www.ti.com/lit/ds/symlink/msp430f1611. pdf, 2011.

[41] Verdult R. and Garcia F. D. and Balasch J. (2012) Gone in 360 Seconds: Hijacking with Hitag2, The 21st USENIX Security Symposium - USENIX Security 2012, USENIX Association, pp. 237-252.

[42] Yang G. and Fan X. and Aagaard M. and Gong G. (2013) Design Space Exploration of the Lightweight Stream Cipher WG-8 for FPGAs and ASICs, The 8th Workshop on Embedded Systems Security (WESS'13), ACM Press, Article No. 8, 2013.

[43] Wu H. and Preneel B. (2005) Chosen IV Attack on Stream Cipher WG, ECRYPT Stream Cipher Project Report 2005/045. Available at http://cr.yp. to/streamciphers/wg/045.pdf. 